\title{
Transferring archival research collections into the digital domain: a special challenge
}

\author{
Christiane FENNESZ-JUHASZ \& Nadja WALLASZKOVITS
}

\begin{abstract}
s
Providing access to research sound collections in the digital domain is a challenge of various kinds. This contribution focuses on the problems related to the digitisation of material archived and structured on analogue archival copies: during the digitisation process it is necessary to go back to the original tapes in order to provide the best technical quality possible. But what does this mean in a practical workflow? Using examples of recent re-archiving projects at the Phonogrammarchiv, the various topics are outlined: starting with the acquisition and analysis of the original tapes, the contribution discusses the problems of re-archiving and segmentation, dealing with differently structured copies, as well as technical and ethical issues as to maintaining integrity and authenticity of the material. The challenge can be handled on the basis of a strong teamwork between curators and technicians.

Die digitale Bereitstellung von Sammlungen wissenschaftlicher Tonaufnahmen stellt für audio-visuelle Archive eine Herausforderung in verschiedener Hinsicht dar. Der vorliegende Beitrag konzentriert sich auf Probleme bei der Digitalisierung von Tonbandaufnahmen, die in strukturierter Form auf analogen Archivkopien gesichert wurden: Um beim Digitalisierungsprozess technisch die bestmögliche Qualität zu gewährleisten, ist es notwendig, auf die betreffenden Originalbänder zurückzugreifen. Doch was bedeutet das für den praktischen Arbeitsablauf? Anhand von Beispielen aus Re-Archivierungsprojekten, die in den letzten Jahren im Phonogrammarchiv durchgeführt wurden, behandelt der Beitrag hier relevante Themen. Beginnend mit der Beschaffung und Analyse der Originalbänder, werden Probleme der Re-Archivierung und Segmentierung, der Umgang mit unterschiedlich strukturierten Kopien sowie technische und ethische Fragen bezüglich der Bewahrung von Integrität und Authentizität der vorliegenden Materialien diskutiert. Diese Herausforderungen sind durch eine enge Kooperation von KuratorInnen und TechnikerInnen bewältigbar.
\end{abstract}

\section{Keywords}

Digitisation, sound recordings, workflow, authenticity, technical and ethical issues

Digitalisierung, Tonaufnahmen, Arbeitsablauf, Authentizität, technische und ethische Fragen

\section{Introduction}

Due to the consistent support policy of the Phonogrammarchiv $(\mathrm{PhA})$ of the Austrian Academy of Sciences (OeAW) since the introduction of magnetic tape technology in the early 1950s, a collection of acoustic research documents came into existence that reflects the essential interest of the 
Austrian research community in the production and analysis of sound recordings, serving as scientific primary sources and, generally, as valuable cultural documents.

Presently, the PhA's holdings comprise a total of ca. 73,000 recordings (i.e. archived items; a total of ca. 13,000 hrs), whereof ca. 46,000 sound recordings (ca. 8,000 hrs) are stored on analogue magnetic tape. Since the beginning of digital archiving in the year 2000, new incoming material as well as archival holdings requested by users have been transferred into the digital domain. However, due to the complexity of the tasks, the lack of additional qualified staff and the amount of daily business for all staff members, a comprehensive systematic retro-digitisation of the original holdings on analogue magnetic tapes has been started only in recent years. The Phonogrammarchiv has meanwhile carried out several tests during the digitisation of selected series and of differently structured material from its tape collection, ${ }^{1}$ and has well defined all the manifold problems involved. Until now, several hundred hours have been digitised and the work is steadily ongoing.

Currently the digitisation of the first 1,000 hrs of the Phonogrammarchiv's tape collection is under way. Core areas of these holdings refer to the systematic documentation of local dialects (free speech, singing) in all Austrian provinces as well as the collection of traditional folk music (including those of minorities, such as the Croats and Hungarians in Burgenland) from 1951 onwards. Fieldwork was conducted by staff members of the archive, in collaboration with researchers from the so-called "Wörterbuchkanzlei" of the OeAW, ${ }^{2}$ folklorists and other experts from university departments, representatives of the "Österreichisches Volksliedwerk" ${ }^{3}$ and also with

1 To date the largest individual series digitised - from the original media, where still available - is a collection of field recordings made between 1961 and 1973 by Károly Gaál (1922-2007, an ethnographer and folklorist, and 1975-1992 a full professor of European Ethnology at the University of Vienna), mainly among the Hungarian, Croatian and German speaking population in Burgenland, Austria's easternmost province; including storytelling, singing and music, and other ethnographic documentation of village life; it comprises ca. $125 \mathrm{hrs}$ and 1,376 archival items respectively.

2 From 1951 up to the mid-1960s, a large-scale project was carried out by the PhA together with Eberhard Kranzmayer, Maria Hornung and Franz Roitinger from the Academy's "Commission for the Creation of the Austrian-Bavarian Dictionary" (later DINAMLEX) in order to collect samples of various Austrian language varieties. The resulting sound recordings include folk tales and other narrations, interviews on ethnographic themes and topical events, etc., and singing. Part of these recordings have already been digitised (ca. 50 hrs / 730 archive numbers so far).

3 Among the researchers who cooperated with the PhA in order to document traditional music were members of the Austrian Folk Song Society such as Karl M. Klier, Georg Kotek, Franz Schunko, Richard Szerelmes, Raimund Zoder and Herbert Lager, as well as the folklorists Hanns Koren, Ludwig Kretzenbacher and Richard Wolfram (cf. Graf 1964). 
local contact persons. In the Phonogrammarchiv's studio samples of foreign languages, other Bavarian dialects, so called "voice portraits" of famous personalities as well as traditional and art music from Austria and abroad were recorded. Another large part of these holdings consists of non-European collections that were created by cultural anthropologists, linguists and ethnomusicologists. After the disruption of Austrian field research activities overseas caused by World War II, these recordings reflect the rise of a young generation of researchers - and other enthusiasts - whose approach to the relevant cultures benefited from being unburdened by colonial history. From 1955 until the early 1960s their expeditions led them to various regions of Northern and sub-Saharan Africa (e.g. Peter Fuchs, Anton Vorbichler, Gerhard Kubik, Andreas Kronenberg, Walter Pichl, Anna Hohenwart-Gerlachstein, Annemarie Schweeger-Hefel) and Madagascar (Lotte Schomerus-Gernböck), to Central Asia and the Indian subcontinent (Max Klimburg/Afghanistan, Hans Strasser/Turkey, Iran, Iraq and Pakistan, Georg Krotkoff/Iraq, Karl Jettmar/Pakistan, René Nebesky-Wojkowitz/Indian Tibetian border region, Heinrich Ketteler as well as Josef Haekel and Engelbert Stiglmayr/Central India), and to South America (Luis T. Laffer/Venezuela). ${ }^{4}$

The Phonogrammarchiv received its first magnetic tape recorder in 1951 (a Philips Maestro, at the time only mains powered), and was able to purchase a second one only in 1954 . However, already before this date, it collaborated with researchers who had carried out fieldwork abroad, and archived their recordings (which had been made with devices lent from third parties). Thus a recording series of Sardinian folk music and ethnographic interviews made by Herbert Melichar, Walter Pöldinger and Umberto Timo in 1953, and songs and narrations recorded in 1954 on the Faroe Islands by Kurt Schier, could be copied shortly after their return from the field. In 1955, the Viennese ethnologist Peter Fuchs was the first to use a batterypowered magnetophone in the field, for his recordings of music of the Tubu in the Tibesti Region (Chad). From 1957 onwards the Phonogrammarchiv supported researchers by equipping them with tape material before their field trips, and it was not until 1958 that it purchased the first three batterypowered tape recorders, which were subsequently used in field work overseas. As a result of this collection policy, which was consequently pursued by Walter Graf (the archive's head since May 1957), the increase of archival holdings (hours of recorded sound) during the second half of the 1950s went up to an annual average that was twelve times higher than in previous years.

4 Further information on the corresponding holdings is available at <http://catalog.pha.oeaw.ac.at/>. 
While archiving, the recording series were comprehensively documented on item level by hand- or type-written "protocols", which also include technical and preservation metadata and are often supplemented by field notes, photographs and other material (such as drawings etc.). This documentation has been already digitised (format: pdf-A). In addition, it was the basis for printed catalogues (Graf \& Hermann 1960, Hermann et al. 1966, Brandl et al. 1970) and content-related metadata capture in the Phonogrammarchiv's in-house database and online catalogue (<http://catalog.pha.oeaw.ac.at/ $>$ ).

\section{Technical and structural background}

Since the PhA was founded, its strategy has been based on the idea of producing sound documents for research purposes under controlled and documented conditions. Therefore, the Phonogrammarchiv has always supported research projects by the loan of carefully selected, technically checked and adequately adapted recording equipment. Due to the various requirements for field recording devices - which should be portable, small in size and light-weighted, ruggedly designed and easy to handle, providing long recording time when battery driven - it has always been necessary to find a compromise between utmost quality and practicality. In the era of magnetic tape recording this meant that portable, battery-driven semiprofessional devices were chosen, which provided the possibility to record on half and quarter track formats and with several speeds, to extend the recording time by switching to a lower speed when needed. In practice, this means that an original tape recorded with such a device can have two or four mono audio tracks, created in opposite directions, on one single tape, sometimes recorded with different speeds (possibly from $2.38 \mathrm{~cm} / \mathrm{s}$ to $19.05 \mathrm{~cm} / \mathrm{s}$ ). One $10 \mathrm{~cm}$ diameter reel can therefore include up to $10 \mathrm{hrs}$ of audio content, recorded with variable parameters.

Once the tapes had been returned to the archive after field research, they were copied to analogue archival tape. To optimise the quality, this was done track by track, in original replay speed, following all the speed switches, and copying one mono (half or sometimes quarter) track to one mono full track, often at a different tape speed. Therefore the archival tape copies offer one perfectly structured mono track in one direction, simply accessible and ready for access. Due to lack of money in the 1950s and 1960 s, in many cases the original tapes were returned to the researcher, while the archive kept the structured archival copies. 
The very first part of the holdings was not even copied, but stored with the original carriers. ${ }^{5}$ Content-related structure was imposed by means of alternately coloured leader tapes. Before 1954, when the Phonogrammarchiv was able to purchase a second reel-to-reel tape recorder, archival copies could be made only in exceptional cases, using the devices of the researchers. Then, due to financial restrictions, it was common practice to return the original tapes to the researchers in lieu of working copies. Thus, one of the main tasks within the digitisation project is to locate those original tapes which were not kept in the Phonogrammarchiv after archival copies had been made.

To turn recordings into useful research sources, careful documentation of every single item and its contents is needed. This work was (and still is) done interactively by sound archivists and field researchers. All contentrelated annotations in the protocols were then carried out following the time references of the archival copies - not the original, potentially differently structured tape.

In many cases, the battery-driven recording devices showed weakness in the stability of power supply and therefore created irregular speed deviations which - during the production of the archival copies - could sometimes be compensated for only insufficiently. Additionally, at the time of producing the early archival copies, there was no adequate technical staff available in the PhA to carry out a high-quality transfer, including routines which are today state-of-the-art, such as individual azimuth alignment, regular machine service and parameter optimisation. Accordingly, a lack in service routines was also related to the portable recording devices. This situation has changed only after the entry of Franz Lechleitner, former (meanwhile retired) chief audio engineer, in the year 1973.

\section{Preservation status}

Part of the holdings have been recorded on or copied to magnetic tapes with a base material of cellulose acetate. Such holdings suffer from a certain risk of degradation, resulting in a loss of information where the existence of adequate high-quality copies to more recent materials is not given.

Generally, audio-visual media with a base material of cellulose acetate are endangered by ageing processes which result in material decomposition. Especially the autocatalytic chemical reaction well known as "vinegar syndrome" (Adelstein et al. 1992) - a hydrolytic process resulting in

5 This was the case, for instance, with most of the PhA's recordings made in cooperation with the Academy's "Wörterbuchkanzlei" (see fn. 2). 
molecular decomposition of the polymer base material, releasing acetic acid and spreading the characteristic smell of vinegar - can lead to the total breakdown of such tapes. Therefore the adequate storage conditions and regular monitoring of devolatilisation of acidity from the material are very efficient preservation measures, which are operated by default in the PhA.

Most recently, a second factor of material damage has been identified: caused by a very slow evaporation of the plasticiser that was used in the production process of magnetic tapes of this early period of time, the material is at risk to become brittle and lose elasticity during long time storage. The Phonogrammarchiv, together with the Austrian Research Institute for Chemistry and Technology OFI, has recently developed a chemical treatment method to permanently refresh the elasticity of such material and to significantly slow down the vinegar syndrome, so that the playability is given for a long time; the method has been patented (OeAW 2011).

Based on the in-house availability of such highly developed preservation measures, the holdings are best prepared to stay in playable condition for further decades. To sum up, the overall preservation status of the early magnetic tape recordings can be regarded as very good or good. Nevertheless, the holdings require constant supervision and ongoing preservation work, also if the contents are to be entirely transferred to digital. The preservation status of tapes still stored outside the PhA at the researchers' homes is however unknown.

\section{Complexity of parameters and equipment obsolescence}

As a most important fact it has to be understood that original tapes, particularly from this period, are not only unique concerning the recorded contents, but are especially unique in their complexity of oftentimes changing technical parameters. Many of the recordings are diverse regarding their signal quality as well as the recording parameters, which quite often are directly related to recording circumstances in the field. In practice, this means that the researcher could be confronted with suboptimal recording locations, either in terms of the acoustic situation or in terms of power and tape supply availability or other adverse circumstances. Therefore it was usual to change parameters such as speed and track format, according to the recording duration that was needed in the field. Sometimes unwanted parameter changes happened by accident. Additionally, to reach long recording times, double and triple play consumer tapes were used in the field along with portable recorders. This means that the tape material by production is only half or one third in thickness. If such tapes are handled on professional standard studio tape machines, which from their default tape tension and winding speed settings are outlined to be used with back-matted 
professional studio tapes (of much higher material stability due to at least double thickness), the thin consumer tapes can be overstretched on spooling and can therefore be irreversibly damaged.

Attention has to be given to the fact that analogue magnetic tape machines for replay are meanwhile nearly out of production. Apart from some exotic and high-priced manufacturers, ${ }^{6}$ even standard studio configurations and parameters can no longer be covered with new professional machines. Obsolescence of high-quality machines that are able to cover the requirements of such demanding materials is therefore a most threatening problem.

Summarising these facts, it is obvious that the adequate high quality digitisation of such holdings can in no case be carried out in form of a mass digitisation transfer, as organised e.g. by radio stations, libraries and other institutions holding technically homogeneous material produced under controlled studio conditions. Thus it appears that such most urgent digitisation cannot be considered a routine sideline job. Well-trained staff with specialised skills in handling and digitising such demanding holdings are necessary to cover the reformatting process from the analogue original to the digital file. In the PhA, this competence of dealing with all kinds of regular and irregular analogue formats has been developed over several decades now, and consequently (also "non-technical") staff members are regularly being trained in this regard.

\section{Strategic considerations}

Regarding the digitisation of the PhA's analogue tape collection, several decisions have already been discussed and tested:

The first decision to be made is whether to digitise from the well-structured archival copies or from the original tapes. As partly outlined above, from a technical point of view, the ultimate signal quality (least noise and distortion, least irregular speed deviations, optimised parameters) can only be reached by using the original tape for transfer into the digital domain. 
Several quality comparisons have impressively confirmed this fact:

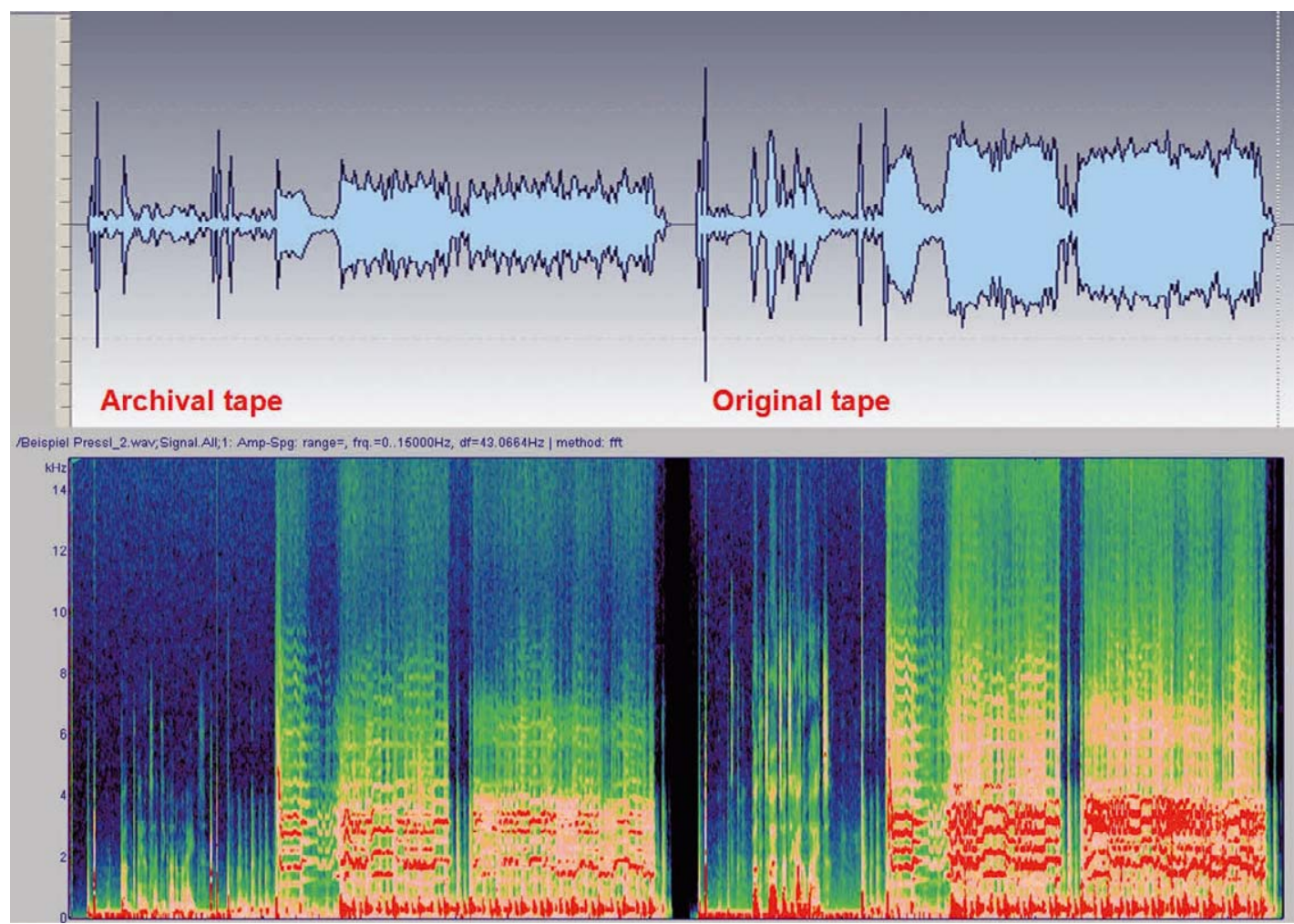

Fig. 1: Waveform (top view) and spectral (bottom view) display of the same recording, as digitised from the archival tape (left side) and the original tape (right side). ${ }^{7}$

Fig. 1 shows the quality comparison between two digitisations, on the left side from the archival copy, and on the right side from the original tape. In both cases the parameters were optimised individually so that both tapes were digitised in the best possible way (individually adjusted azimuth, level, correctly selected track width, etc.). It is obvious that due to the generation

7 Source: The first 15 seconds of PhA B 13279, recorded on 14 November 1968 by H. M. PreßI in Mazar-i-Sharif (Balkh Province, Afghanistan), including a spoken announcement, noise of on-off switches and from sec 5 onwards dul and sornai (drum and shawm) playing by Uzbek musicians from Karisimir near Kabul. The comparison also reveals that, obviously after archiving, the announcement at the beginning was recorded for a second time on original tape to replace the erroneous original one ("Band 21 a Nummer 1" on archival tape vs. "Band 22 a Nummer 1" on original tape). - The example belongs to a series of music recordings made by the Austrian composer and music teacher Hermann Markus PreßI (1939-1994) between 1967 and 1970 in various regions of Afghanistan; the collection has recently been digitised by using the originals (ca. $38 \mathrm{hrs}, 531$ archive nos.). Spectrograms performed by analysis software S_TOOLS-STx, Institute of Acoustic Research of the Austrian Academy of Sciences, Vienna. 
loss between copy and original and the additional high frequency loss due to azimuth mismatches, the analysis of the copy shows spectral signal components up to only $10 \mathrm{kHz}$, while on digitisation from the original, the full brightness of the recording with spectral components above $15 \mathrm{kHz}$ is clearly visible.

Aside from the advantage in signal spectrum, level and brightness, other parameters that are not so clearly analysable (e.g. distortion, speed irregularities, noise, etc.) sum up and determine the overall signal quality of the digitisation. Therefore the much better signal quality is a convincing argument, and is certainly recommended in the guidelines (IASA-TC 03, ch.5, and others).

As outlined, in many cases the original tapes differ from the archival copies in structure as well as in recording duration, due to the fact that not all items recorded have necessarily been kept and copied to archival tape. In several cases the researcher decided not to archive certain items, for whatever reason. In such cases the original tape carries more contents than the archival copy. But surprisingly, sometimes the original tapes can contain less content than the archival tapes, due to accidental erasure or even wanted out-cuts/restructurings carried out by the researcher. Additionally, since the content-related documentation refers to the often differently structured archival copies, it is necessary to digitise both, the original recordings as well as their archival copies, in parallel.

Therefore, in a second step of the workflow, the content and the time relation between both digitisations have to be matched and finally harmonised with the archival documentation before being captured in the database.

The procedures just described are, of course, very time-consuming and for the most part of the collection - cannot be automated. In the worst case, an accurate digitisation of one single original tape can take up to 10-15 hrs of transfer and editing, and an average time of $3 \mathrm{hrs}$ or - in the case of two existing analogue versions -5 hrs per hour of original content can be realistically calculated for the technical work, potential content-matching of the two digitised versions, editing of the new archival master, metadata alignment with the existing documentation and capture of transfer metadata in the database.

Thus, one could argue for storing both digitised versions "un-matched" and not edited, only with cursory cross-references in the technical documentation, and adjourn the detailed matching and correct structuring of the digital archival masters (gained from the original tapes) indefinitely, by refraining from this undertaking until the time of the next request for use of these archival holdings. However, our experiences have shown that such 
a procedure is not practicable especially when one is dealing with larger series of field recordings that span longer time periods. Furthermore, we think that - due to today's reasonable user expectations regarding direct and quick access - in those cases where digitised versions of archival and original tapes exhibit considerable differences, the already structured one gained from the archival tape, although of poorer signal quality, will be accessed. In addition, in some cases efficient matching can be carried out only with a general overview of a whole recording series and by using additional information available, such as tape splices and notes on original carriers, their reels or containers; finally, as a matter of principle, it shall be traceable and therefore well documented. Such work can be done most economically by the staff in charge during the digitisation process, but not at a later point by using the digitised representations of recordings and information carriers only.

The arguments outlined here can be well illustrated by various - sometimes unexpected - instances which our co-workers and we have come across during the past years. Károly Gaál's extensive collection may serve as an example (see fn. 1): the 101 tapes still available in his legacy ${ }^{8}$ include 145 hrs of sound (recorded half track); however, at least 15 of the originals - which are serially numbered on their reels and (mostly additionally labelled) containers - are missing. While nearly all other carriers include segments (of some seconds up to whole tracks of ca. $1 \mathrm{~h}$ ) that had not been copied before, and six turned out to be complete copies (mainly of already archived material, and broadcast or commercial recordings), another eight tapes could not be allocated at all, because their entire content ${ }^{9}$ had not been archived or had replaced the original one after its archiving (as was also the case with some of the copies just mentioned). Matching procedures became even more complicated due to the fact that some tapes had been re-numbered after archiving, and eight original reels had later been exchanged for each other (and then numbered according to the information on the wrong container). In addition, some - fortunately only seven - original tapes featured short discontinuities or defects, such as accidental erasures (mostly of some seconds only), sporadic tape breaks and other damages (e.g. overexpansion), or speed fluctuation, so that the corresponding sections of the recordings had to be replaced by their counterparts from

8 Due to a donation made as early as 1986, part of this estate (including these tapes) is now housed in the Savaria museum of Vas County in Szombathely, Hungary.

9 These tapes include mostly interviews and other background information on archived contents, partly recorded at a later date. 
archive tapes. Finally, 183 (i.e. ca. 20 hrs) of the 1,376 archival items had to be taken from the digitised archival copies.
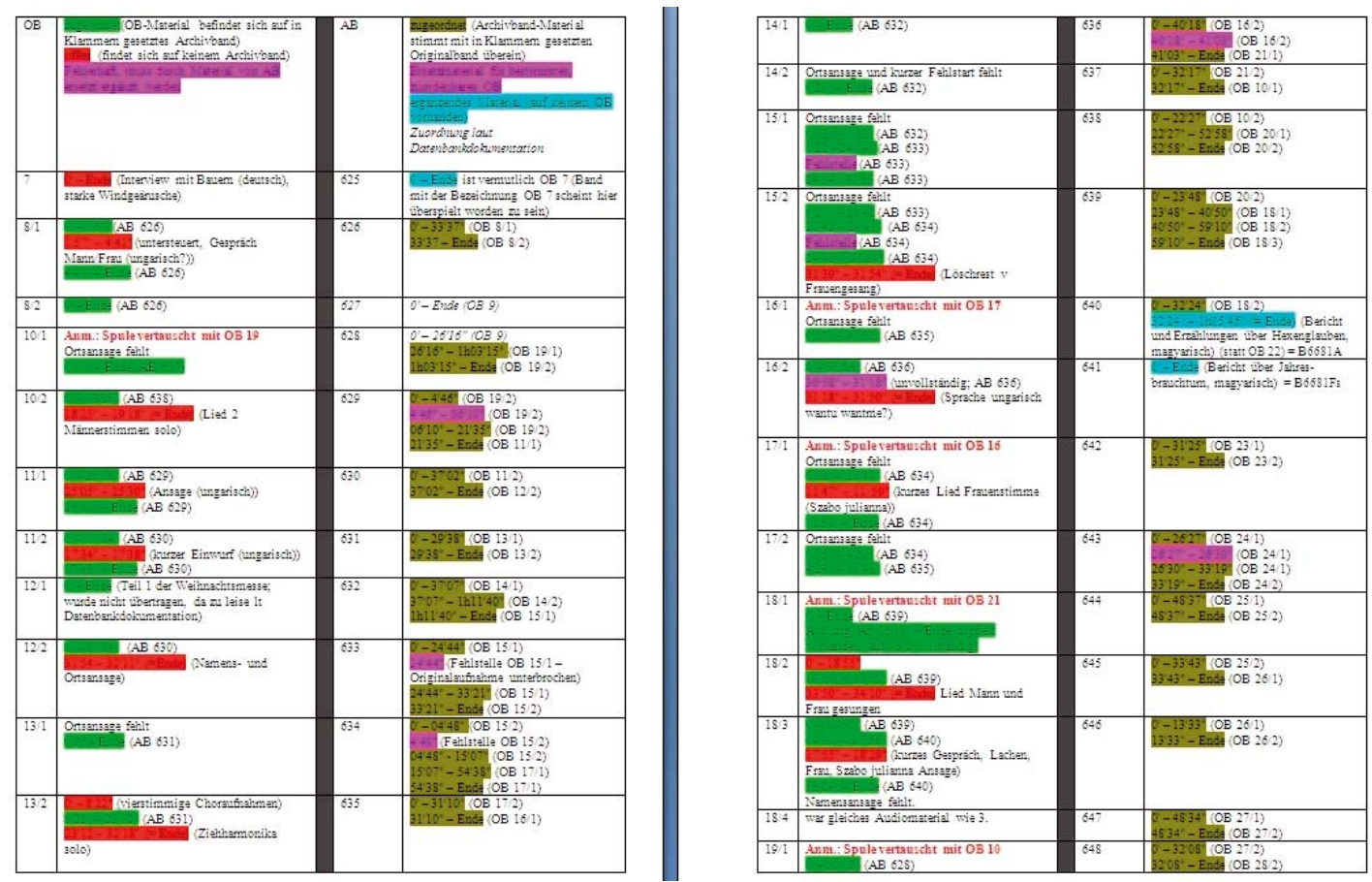

Fig. 2: The first two pages of a matching-help list written by Maria Födisch during the digitisation of the Gaál collection.

Fig. 2 illustrates the sometimes challenging process of allocating original recordings to analogue archival copies. The left half of a page (two columns) refers to original tapes $(\mathrm{OB})$ of the Gaál collection, the right half to archival tapes (AB). - Legend of colours on the time references: green = section of $O B$ matched with section of $A B$ [tape no. written in brackets]; red = section of $O B$ not detectable on any $A B$; pink = defective part of $O B$, replaced by material from $A B$; blue = section of $A B$ not detectable on any $O B$; olive = section of $A B$ matched with section of $O B$ [tape no. and track in brackets]; time reference of $A B$ in italics, not coloured = material substituting a missing (section of an) OB.

In our opinion, the described loss, confusion or re-use of tapes is quite characteristic for large collections that cover longer time spans and have served the researchers as working copies over many years (for transcription, analysis, educational usage, etc.).

Another example is the legacy of Alfred Jilka (1921-2005), a professional Austrian violinist and enthusiastic ornithologist specialising in domestic bird sounds. This series of 86 tapes (with a total duration of $36 \mathrm{hrs,} \mathrm{recorded}$ 
1964-1983) included also the original carriers of recordings from 19661969 that had already been archived on magnetic tape (4.5 hrs, 62 archive nos.). However, during digitisation it turned out that four of these 11 original tapes included additional recordings of bird songs, with - even more tricky - eight of them later edited by means of cutting out parts; thus, 12 digitised original recordings did not match their analogue archival representations because they featured - partly several - discontinuities of some seconds up to 3.5 minutes. Of course, these divergences of the archival copies and the originals were mostly evident due to considerably differing signal durations; however, since the researcher's otherwise accurate original documentation (which refers to the new "masters") lacks notes on the cutting procedures, exactly locating the out-cuts is only possible by inspection of the original tapes and precise (aural) comparison of both versions. Whatever decision will be made when dealing with such recordings - whether to save both digitised versions, or create a new digital item by collating them (as in the present case) - all processing has to be clearly annotated in the corresponding documentation in order to be reproducible. ${ }^{10}$

An additional benefit of and another argument for consistent digital "rearchiving" of each single item is the detection and correction of errors which happened during the archiving on magnetic tape. Generally, such mistakes are rather rare, and most of them concern the written documentation. Typical mistakes in writing are wrong durations of items on archival tapes (e.g. $5 \mathrm{~m} 45 \mathrm{~s}$ instead of correct $2 \mathrm{~m} 55 \mathrm{~s}$ ) or - more rarely - wrong number labelling of successive archive tapes, their reels and containers. More surprisingly, but fortunately hardly ever appearing, are cases of wrong structuring of archive tapes and concurrent erroneous documentation. So far, we have only twice come across numbered and annotated archival items that were not to be found, neither on archival nor on original tape, and thus in fact do not exist; and it was only on one occasion that the two half tracks of an original tape, each including the continuation of tales interrupted by tape/ track change, had been mixed up during analogue archiving so that wrong

10 In this special case, one might say, we deal "only" with some moments of - in principle selected - acoustic excerpts of the natural environment, thus the versions later cut by the recordist could be treated as the new archival items. Apart from the fact that such arguments apply to any media representation, the reasons for the manipulations mentioned remain, however, unclear, and divergences between the existing versions have to be documented in any case. Moreover, sound archivists have to act according to professional ethical principles, which include clear guidelines for the preservation of archival recordings, such as the following: "Their original content and physical representation shall be safeguarded from being modified, truncated, extended, falsified or censored in any way." (Assmann et al. 2011: ch. 2.2.1). 
parts of contents were grouped, and accordingly incorrectly annotated. ${ }^{11}$

Such rare errors are, of course, absolutely understandable, especially if the archiving procedures of analogue material are taken into consideration; possible reasons might be: errors while typing notes that were written by hand during the copying (in real time!), potential interruption of work which is then continued at a later date, necessary rewinding for continuing or control listening, or selecting a leader tape with the wrong colour.

Finally, another positive side effect of this undertaking is worth mentioning. In our experience, after thorough instruction and with constant supervision by specialised staff members, digitisation and matching procedures can also be carried out by young co-workers (freelancers, students or trainees); it can thus serve as a hands-on training for individuals who have grown up entirely in the digital age and generally have little, if any, practical experience with analogue media and their characteristics and archiving. This sort of oral tradition guarantees the best possible knowledge transfer in practical handling, which otherwise would have to be collected from rare written sources or - in as yet undocumented cases - would be totally lost. In addition, while completing and capturing the relevant metadata, the basics of technical and content-related archival documentation are learnt, and a routine in doing so is gained.

Getting back to the specific technical challenges of the holdings in question, another problem could occasionally arise during digitisation: for the recording process portable consumer recorders were used, which can show significant irregular speed deviations, e.g. due to problems with tape transport in the field, or caused by problems of low battery charge. For several years, the Phonogrammarchiv has been carrying out substantial research in using the high frequency bias signal recorded on every magnetic tape as a reference for speed fluctuations and their correction in the analogue or digital domain (cf. Wallaszkovits et al. 2014); where applicable, this method will be used as well.

\section{Specific methods}

Digitisation is carried out following highest international quality guidelines and best practices, above all the de-facto standards IASA-TC 03 and IASA-TC 04 (publications that were co-authored by staff members of the

11 The two absent archival items (from the Preßl collection, see fn. 7) are B 13082 and B 13408, the protocols list them with their duration ( $1 \mathrm{~m} 42 \mathrm{~s}$ and $3 \mathrm{~min} 35 \mathrm{~s}$ ) and reference to the corresponding (though not existing) recording on the original tape; the wrong end of B 9661 on the archive tape is actually the continuation of B 9675, while the correct end of the tale was then individually numbered B 9670 (all items from the Gaál collection). 
Phonogrammarchiv) as well as Sound Directions: Best Practices for Audio Preservation (Casey \& Gordon 2007) and other relevant publications (Boston 1998, Copeland 2008). The use of regularly maintained and fully checked state-of-the-art equipment and components in all steps of the digitisation workflow is obvious. Where applicable, the chemical treatment method will be utilised (e.g. as preventive and quality rising measure), as well as the method to capture the bias signal as speed reference, to guarantee the best possible audio signal quality.

Basic archival workflow

The digitisation workflow for both, the originals as well as the archival copies, is carried out according to the following steps:

- Analysis of the preservation status of the individual tape

- Preparation of the tape for transfer:

- physical restoration where applicable (e.g. repairing bad/bleeding splices), adding leader tape where necessary (e.g. for capturing contents starting at the very beginning of the tape)

- chemical restoration where applicable (e.g. permanent refreshment of the elasticity of acetate tapes)

- cleaning of the tape if necessary

- multiple rewinding processes of every single tape to reduce storagerelated artefacts (e.g. print-through effect, cf. Bertram et al. 1980)

- Preparation of the tape machine for transfer:

- transfer on regularly maintained and cleaned tape machines according to specifications (e.g. IASA-TC 04)

- choice of correct replay parameters for every single tape (speed, track format, equalisation)

- individual azimuth alignment for every single tape

- bias read out if necessary / where applicable for correction of irregular speed fluctuations

- Digitisation of the recordings in WAVE file format, $24 \mathrm{Bit} / 96 \mathrm{kHz}$ resolution (or higher, if special restoration needs are expected), as archival master

- Creation of transfer metadata in the database of the Phonogrammarchiv, documenting all restoration needs and technical parameters

- Creation of user copies in access format (MP3) 


\section{Metadata matching workflow}

Matching procedures start already during digitisation, by a virtual segmentation of the wave file using the marker tool of the audio editor program. This is an easy and fast task with archival tapes because, due to the added coloured leader tapes, there is a minimum of 15 seconds silence each between the individual archival recordings included. In this case, the presegmentation can also be carried out by applying the automatic auto-split routine of the audio editor on the saved sound file (with subsequent manual correction). In a next step, the corresponding segments of the digitised track(s) of the relevant original tape(s) are identified by comparison with the digitised archival versions (and by accessing the correspondent protocols), and pre-segmentation is carried out by manually setting markers of the audio editor. During this step, it is checked whether the marked segments in the two versions totally overlap or whether one contains parts that are missing in the corresponding other. Subsequently, the individual archival items, named with the original identifiers (plus D) and including a start- and an end-marker, are saved from the digitised file (which remains uncut). If during the alignment procedure absent or defective parts in the original version are detected, these are replaced by the corresponding segments of the digitised archival version.

The digitised items must then be linked to the archival documentation. This is done by entering the names of the wave files (and the MP3 access copies), their durations, original tape, headroom, etc. in the "numbers" section of the correspondent data sets; in order to make the recordings callable via the database directly from the server, there is also a "marker" at the starting time reference of each item, created by using a special plug-in of the relevant editor field. To speed up this procedure, a short script ${ }^{12}$ converts the time references and content description (here the archive no.) contained in the marker files of the audio editor into a format (HTML) that can be copied directly into the relevant database field without the need of using the editor plug-in. In addition, working procedures can be rationalised in the case of only one available version (the cut archival tape with the original recordings): the new digital masters are created here in correspondence with the archival (and database) structure, which groups individual items of a recording session; thus, where possible, wave files including a series of successive archival numbers (each with a marker at its beginning and end) are saved and accordingly entered as a collective number in the database (by using the mentioned HTML script for listing the items with recallable time references).

12 The script was written by Friedrich Neubarth (Vienna) using the open programming language Perl. 
Further tasks here include the correction of potential errors in the original archival documentation and/or during analogue archiving (as described above), and finally a summary documentation of additional - but formerly not archived - contents of original tapes. This documentation is saved in the Material section of the digital archive, where also other digitised information (scanned reel/carrier labelling, field notes, etc.) is stored and accessible via the database. ${ }^{13}$

\section{Conclusion}

Transferring archival research recordings from the analogue into the digital domain is a necessary and rewarding undertaking that guarantees their sustainable preservation and quick accessibility; when conducted according to state-of-the-art standards, however, it turns out to be a labour intensive project. As has been shown, the reformatting process from the analogue originals to digital files cannot be carried out as an automated procedure, but involves various processes and methods to be performed individually. For potentially economising some procedures outlined in this paper, the development of automatic identification and alignment methods for corresponding sound segments is currently observed, and tests are planned in how far these methods ${ }^{14}$ are already applicable to ethnographic field recordings (of heterogeneous contents and varying audio quality). In any case, it is the archive's responsibility to conduct all necessary tasks in order to provide easy availability in best possible quality of these research sources and representations of cultural heritage.

13 The additional original recordings stay available in the uncut digitised wave files (at the end stored "near line"), for a more detailed inspection and possible archiving at a later stage; this is best decided together with the researchers or performers, if still available, or other experts, but in any case individually per item.

14 These methods include audio fingerprinting (obtained from spectral analysis) and audio similarity estimation methods for music recordings, or formant extraction, pitch detection etc. for analysis of speech (cf. Sonnleitner \& Widmer 2014; Schlüter 2013; Six \& Cornelis 2012; Grachten et al. 2013; Sonnleitner et al. 2012). 


\section{References}

Adelstein, P.Z., J.M. Reilly, D.W. Nishimura \& C.J. Erbland. 1992. "Stability of Cellulose Ester Base Photographic Film: Part I-Laboratory Testing Procedures". SMPTE Journal 101 (5): 336-346.

Assmann, Ilse et al. 2011. Ethical Principles for Sound and Audiovisual Archives. (IASA Special Publication No. 6). Rev. ed. <http://www.iasa-web.org/ethical-principles> (20/6/2015).

Bertram, Neal, Michael K. Stafford \& David R. Mills. 1980. "The Print-Through Phenomenon". Journal of the Audio Engineering Society (JAES) 28 (10): 690-705.

Boston, George (ed.). 1998. Safeguarding the Documentary Heritage: A guide to Standards, Recommended Practices and Reference Literature Related to the Preservation of Documents of All Kinds. Paris: UNESCO. Online: <http://www.unesco.org/webworld/mdm/administ/en/guide/guidetoc.htm> (20/6/2015).

Brandl, Rudolf, Elisabeth Deutsch, Werner Deutsch \& Dietrich Schüller. 1970. Katalog der Tonbandaufnahmen B 7001 - B 10000. (84. Mitteilung der Phonogrammarchivs-Kommission der Österreichischen Akademie der Wissenschaften). Wien: H. Böhlaus Nachf.

Casey, Mike \& Bruce Gordon (eds.). 2007. Sound Directions: Best Practices for Audio Preservation. Online: <http://www.dlib.indiana.edu/projects/sounddirections/papersPresent/sd_bp_07.pdf> (20/6/2015).

Copeland, Peter. 2008. Manual of analogue sound restoration techniques. London: British Library.

Grachten, Maarten, Martin Gasser, Gerhard Widmer \& Andreas Arzt. 2013. "Automatic alignment of music performances with structural differences". Proceedings of the $14^{\text {th }}$ International Society for Music Information Retrieval Conference (ISMIR 2013; Curitiba, Brazil): 607-612.

Graf, Walter. 1964. "Aus der Geschichte des Phonogrammarchivs der Österreichischen Akademie der Wissenschaften". Biuletyn fonograficzny / Bulletin phonographique VI: 9-39.

Graf, Walter \& Elfriede Hermann. 1960. Katalog der Tonbandaufnahmen B 1 - B 3000. (81. Mitteilung der Phonogrammarchivs-Kommission der Österreichischen Akademie der Wissenschaften). Wien: H. Böhlaus Nachf.

Hermann, Elfriede, Herbert Schendl \& Dietrich Schüller. 1966. Katalog der Tonbandaufnahmen B 3001 $-B$ 7000. (82. Mitteilung der Phonogrammarchivs-Kommission der Österreichischen Akademie der Wissenschaften). Wien: H. Böhlaus Nachf.

IASA-TC 03 = Schüller, Dietrich (ed.). 2005. The safeguarding of the Audio Heritage: Ethics, Principles and Preservation Strategy. Version 3, December 2005. (IASA Technical Committee: Standards, Recommended Practices and Strategies; IASA-TC 03). [Budapest]: IASA. Online: <http://www.iasa-web.org/ sites/default/files/downloads/publications/TC03_English.pdf> (20/6/2015).

IASA-TC 04 = Bradley, Kevin (ed.). 2009. Guidelines on the Production and Preservation of Digital Audio Objects. Second edition. (IASA Technical Committee: Standards, Recommended Practices and Strategies, IASA-TC 04). [Auckland Park]: IASA. Online: <http://www.iasa-web.org/audio-preservation-tc04> $(20 / 6 / 2015)$.

OeAW. 2011. Österreichische Akademie der Wissenschaften: Method for Reconditioning Data Carriers. Inventors: P. Liepert, L. Spoljaric-Lukacic, N. Wallaszkovits. Appl. filed 23.12.2011, published 05.07.2012. IPC: G11B 23/50 (2006.01), G03D 15/00 (2006.01). Pub. No.: WO/2012/0885.

Schlüter, Jan. 2013. "Learning Binary Codes for Efficient Large-Scale Music Similarity Search". Proceedings of the 14th International Society for Music Information Retrieval Conference (ISMIR 2013; Curitiba, Brazil): 581-586. 
Six, Joren \& Olmo Cornelis. 2012. "A robust audio fingerprinter based on pitch class histograms: applications for ethnic music archives". In: Díaz Báñez, J. M., F. J. Escobar Borrego \& I. Ventura Molina (eds.). Boundaries between Genres: Flamenco and Other Musical Oral Traditions. III Interdisciplinary Conference on Flamenco Research - INFLA. II International Workshop of Folk Music Analysis - FMA. Sevilla, April 19th-20th 2012. Sevilla: Escuela Técnica Superior de Ingeniería, 191-198. Online: <http://congreso. us.es/infla/infla2012/documents/Book-INFLA_FMA.pdf> (20/6/2015).

Sonnleitner, Reinhard \& Gerhard Widmer. 2014. “Quad-Based Audio Fingerprinting Robust To Time And Frequency Scaling". Proceedings of the $17^{\text {th }}$ International Conference on Digital Audio Effects (DAFx-14), Erlangen, Germany, September 1-5, 2014. <http://www.dafx14.fau.de/papers/dafx14_reinhard_sonnleitner_quad_based_audio_fingerpr.pdf> (30/11/2015).

Sonnleitner, Reinhard, Bernhard Niedermayer, Gerhard Widmer \& Jan Schlüter. 2012. "A Simple and Effective Spectral Feature for Speech Detection in Mixed Audio Signals". Proceedings of the $15^{\text {th }}$ International Conference on Digital Audio Effects (DAFx-12); York, UK, September 17-21, 2012. Online: <http:// dafx12.york.ac.uk/papers/dafx12_submission_19.pdf> (20/6/2015).

Wallaszkovits, Nadja, Tobias Hetzer \& Heinrich Pichler. 2014. "Drift and Wow Correction of Analogue Magnetic Tape Recordings in the Analogue Domain Using HF-Bias Signals". Proceedings of the $136^{\text {th }}$ AES Convention (26-29 April 2014, Berlin, Germany; Convention Paper 9042): 1-10.

\section{Christiane FENNESZ-JUHASZ: \\ Phonogrammarchiv - Austrian Academy of Sciences \\ http://www.phonogrammarchiv.at/wwwnew/index_e.htm \\ christiane.fennesz-juhasz@oeaw.ac.at}

Nadja WALLASZKOVITS:

Phonogrammarchiv - Austrian Academy of Sciences

http://www.phonogrammarchiv.at/wwwnew/index_e.htm

nadja.wallaszkovits@oeaw.ac.at

doi: 10.1553/jpa6s126-143 